\title{
Ictiofauna capturada por pescadores artesanais na Lagoa Pequena - Região estuarina da Lagoa dos Patos - RS
}

\author{
Clarissa Alves ${ }^{1}$ \\ Fabiano Corrêa ${ }^{1}$ \\ Alex Bager ${ }^{2}$ \\ Juvêncio Pouey Luís Osório Fernandes ${ }^{3}$ \\ Sérgio Renato Noguez Piedras Piedras ${ }^{3 *}$ \\ ${ }^{1}$ Universidade Católica de Pelotas, Pelotas - RS, Brasil \\ ${ }^{2}$ Universidade Federal de Lavras - MG, Brasil \\ ${ }^{3}$ Laboratório de Ictiologia, Departamento de Zootecnia, FAEM \\ Universidade Federal de Pelotas, Pelotas - RS, Brasil \\ Campus Universitário do Capão do Leão, FAEM/UFPel \\ Caixa Postal 354, CEP 96010-970, Pelotas - RS, Brasil \\ *Autor para correspondência \\ sergio.piedras@ufpel.tche.br
}

\section{Resumo}

Através de avaliações mensais foram identificadas as principais espécies de peixes capturadas por pescadores artesanais que atuam na Lagoa Pequena, localizada na região estuarina da Lagoa dos Patos - RS. Os dados de biomassa, freqüência absoluta e relativa de cada espécie foram submetidas à análise de correlação com a estação do ano e variáveis ambientais. Embora a salinidade tenha se mantido acima de 3,5, houve predominância da captura de $85,5 \%$ de espécies características de água doce e $15,5 \%$ de espécies estuarinas. O valor econômico das espécies, as artes de pesca e estação do ano são determinantes na produção pesqueira local.

Unitermos: espécies de peixe, estuário, pesca artesanal, salinidade

\section{Abstract}

Ictiotiofauna captured by artisan fishermen in Lagoa Pequena - Estuarine region of Lagoa dos Patos - RS. Through monthly evaluations, the main species of fish captured by the fisherman of Lagoa Pequena, a site in the estuarine region of Lagoa dos Patos - RS, were identified. The biomass data, in addition to the absolute and relative frequencies of each species, were submitted to correlation analysis with the seasons of the year and environmental variables. Although water salinity was found to be constantly above 3.5 , there was a predominance of the capture of $85.8 \%$ of characteristic freshwater species and $15.5 \%$ of estuarine species. The economic values of the species, the types of fishing and the seasons of the year are the main determinants of the local fishing production.

Key words: artisanal fishing, estuary, salinity, species of fish 
Nos estuários, existe uma grande biomassa de peixes associada à alta produtividade primária desses ambientes (Loebmann et al., 2008), o que lhe assegura uma importante função social e econômica para as comunidades que vivem em seus arredores, onde são encontrados muitos pescadores artesanais. $\mathrm{O}$ estuário da Lagoa dos Patos é uma região de encontro entre as águas provenientes de uma bacia de drenagem de aproximadamente $200.000 \mathrm{~km}^{2}$ e as águas costeiras da margem oeste do Oceano Atlântico Sul. Ele ocupa em torno $10 \%$ da superfície total da Lagoa dos Patos, sendo caracterizado por amplas regiões de bancos $(80 \%)$, canais naturais e artificiais e sacos marginais rasos.

A Lagoa Pequena é um ambiente natural com uma superfície de 4.000 ha. Localiza-se entre os paralelos $31^{\circ} 33^{\prime}$ e $31^{\circ} 18^{\prime}$ de latitude sul e $52^{\circ} 02^{\prime}$ e $52^{\circ} 06^{\prime}$ de longitude oeste, inunda terras dos municípios de Pelotas e Turuçu, no sul do Rio Grande do Sul. Atualmente seus usos predominantes são: utilização da água para irrigação dos cultivos do arroz, quando não esta salinizada e pesca.

Através de canais naturais de ligação com a Lagoa dos Patos, a Lagoa Pequena sofre influência tanto físico-química, como biológica da zona estuariana da Lagoa dos Patos, tornando-a parte integrante do sistema estuarial e diferenciando-a de um corpo de água essencialmente límnico.

Em relação à fauna ictiológica da região estuarina, onde esta inserida a Lagoa Pequena, Garcia e Vieira (2001) classificam as populações como residentes, aquelas que têm ciclo de vida completo no estuário; espécies estuarinas dependentes são aquelas que desovam no mar ou estuário e têm as fases iniciais de vida, desenvolvidas no estuário; espécies marinhas visitantes são aquelas de vida exclusivamente marinha com raras incursões no estuário; e as espécies visitantes de água-doce, as quais são características de ambientes límnicos e fluvial, só ocorrendo no estuário em períodos de cheias, quando a salinidade é reduzida.

Devido sua proximidade com a Colônia de pescadores Z-3 de Pelotas, a Lagoa Pequena é usada como local de pesca, por um grupo de 30 a 50 parelhas (grupo de 2 a 3 pescadores em uma embarcação de pequeno porte e equipamentos de pesca), de acordo com a época do ano (ITEPA, 2002). Estando incluída na região estuarina a pesca local é regulada pela Instrução Normativa conjunta $\mathrm{n}^{\circ} 03$ de 09/02/2004 do Ministério do Meio Ambiente e Secretária Especial de Aqüicultura e Pesca, que determina os períodos e equipamentos de pesca permitidos, para as espécies estuarino dependentes e marinhas visitantes, mas não se refere à pesca de espécies exclusivamente de água doce, que de acordo com os pescadores locais, são objeto principal da atividade pesqueira local.

Súarez e Junior (2005) e Súarez (2008) estudando a composição e distribuição de espécies de peixes em regiões neotropicais, afirmam que são poucos os estudos e mesmo listagem de espécies com ocorrência nestes ambientes são escassas. Assim o objetivo desse estudo é identificar a ictiofauna capturada por pescadores artesanais que atuam na Lagoa Pequena, como informação básica para gestão da pesca local.

Entre os meses de março de 2005 e fevereiro de 2006 foram realizadas amostragens uma vez ao mês, nas quais utilizando uma lancha rápida abordaram-se aleatoriamente, três barcos de pesca que atuam na lagoa, totalizando 36 amostragens. Do total capturado por cada embarcação, no final da pescaria, foram subtraídas amostras aleatórias das espécies capturadas. Para capturas de biomassa até cinco quilos de cada espécie, foi coletado um exemplar, quando a biomassa de uma determinada espécie capturada foi superior a $20 \mathrm{~kg}$, coletaram-se quatro exemplares.

A pesca na Lagoa Pequena se caracteriza pelo uso de embarcações de pequeno porte, com capacidade de 4.000 a $6.000 \mathrm{~kg}$, em que atuam dois ou três homens que uso redes de emalhe com malhas entre 70 e $100 \mathrm{~mm}$ entre ângulos opostos, que são revisadas diariamente quando o peixe capturado é recolhido. O numero de redes é variável entre as embarcações, de maneira que foi considerado esforço de pesca no estudo.

Os animais coletados de cada embarcação, separados por espécie, foram levados ao laboratório de Ictiologia da Universidade Federal de Pelotas, onde foram identificados de acordo com Fischer et al. (2004) e Bemvenuti e Moresco (2005), após os exemplares 
foram acondicionados em álcool 70\% e depositados na coleção de peixes do Laboratório de Limnologia a da Universidade Católica de Pelotas.

As variáveis de temperatura da água e salinidade foram medidas nos locais de abordagem das embarcações. Os índices pluviométricos foram medidos com pluviômetro, localizado em propriedade rural junto à margem oeste da Lagoa Pequena.

Todos os animais foram medidos em comprimento total, com ictiometro e peso total em balança digital. Foi determinada a biomassa, a riqueza de espécies, a freqüência absoluta e relativa de cada espécie. Os resultados foram agrupados por estação do ano, sendo janeiro, fevereiro e março os meses considerados para o verão; abril, maio e junho os meses de outono; julho, agosto e setembro os meses de inverno; e outubro, novembro e dezembro os meses de primavera. Os dados ambientais, por estação do ano, foram avaliados pelo teste ANOVA, e quando resultou diferença significativa as médias foram comparadas pelo de Duncan $(\mathrm{P} \leq 0,05)$. Os dados de freqüência de ocorrência das espécies capturadas e as variáveis ambientais foram agrupados por estação do ano e submetidos à análise de correlação de Pearson $(\mathrm{P} \leq 0,05)$.

A temperatura da água apresentou variação significativa caracterizando dois períodos no ano. $\mathrm{O}$ período de primavera e verão, com médias de $22,6^{\circ} \mathrm{C}$ e $24,2^{\circ} \mathrm{C}$, e outono e inverno com médias de $13,8^{\circ} \mathrm{C}$ e $16.6^{\circ} \mathrm{C}$, respectivamente (Tabela 1 ).

TABELA 1: Médias e desvio padrão das variáveis ambientais e riqueza de espécies na Lagoa Pequena entre março de 2005 a fevereiro de 2006.

\begin{tabular}{lccccc}
\hline $\begin{array}{l}\text { Estação/ } \\
\text { Variável }\end{array}$ & Outono & Inverno & Primavera & Verão & D. P. \\
\hline $\begin{array}{l}\text { Temperatura } \\
\left({ }^{\circ} \mathrm{C}\right)\end{array}$ & $16,6_{\mathrm{b}}$ & $13,8_{\mathrm{b}}$ & $22,6_{\mathrm{a}}$ & $24,2_{\mathrm{a}}$ & 2,52 \\
$\begin{array}{l}\text { Salinidade } \\
\begin{array}{l}\text { Precipitação } \\
(\mathrm{mm})\end{array}\end{array}$ & $91_{\mathrm{a}}$ & $7_{\mathrm{b}}$ & $35_{\mathrm{b}}$ & $10_{\mathrm{a}}$ & 2,15 \\
\hline
\end{tabular}

Letras diferentes na mesma linha indicam diferença significativa pelo teste de Duncan $(\mathrm{P} \leq 0,05)$.
A salinidade variou entre 3,5 e 11 na superfície, e sendo esta variação devido a precipitação pluviométrica ocorrida na região, que apenas na primavera manteve-se nos níveis normais, acima dos $110 \mathrm{~mm}$, de acordo com a Estação Agroclimatologia da UFPEL.

Durante o estudo foram coletados 330 indivíduos, pertencentes a cinco ordens, 11 famílias e 15 espécies. Os Characiformes (Cyphocarax voga, Hoplias malabaricus, Ologossarcus jenynsii, O. robustus) predominaram com 41,5\% seguidos de Siluriformes (Hypostomus comersonii, Loricariichthys anus, Pimelodus maculatus, Rhamdia aff. quelen,) com 26,4\%, de Perciformes (Crenicichla punctata, Geophagus brasiliensis, Micropogonias furnieri) com 19,7\%, os Atheriniformes (Odontesthes aregentinensis, $O$. bonariensis) com $8,8 \%$, Mugiliformes (Mugil platanus.) com 5,5\% e Pleuronectiformes (Paralichthys orbignyanus) com 3,6\% (Tabela 2).

A predominância na captura de espécies de água doce que representaram mais de $84,5 \%$ da freqüência de ocorrência caracteriza a pesca local, diferente da que ocorre na região lagunar, onde atuam a maioria dos pescadores da Colônia Z-3, e nas capturas predominam as espécies marinhas e estuarinos dependentes (Garcia e Vieira, 2001; Bemvenuti e Moresco, 2005).

A análise da sazonalidade demonstrou um maior percentual de captura na primavera-verão, com $64,2 \%$ em contra 35,8\% no outono-inverno. Esta diferença é explicada pela maior abundância de espécies na primavera-verão (Tabela 2), que por sua vez, pode ser atribuída as maiores temperaturas e salinidades registradas no período, favorecendo o deslocamento da maioria das espécies para alimentação e reprodução, conforme o registrado por Cerdeira et al. (2000) que, estudando a pesca no baixo Amazonas afirmam que variações ambientais favorecem migrações para reprodução e alimentação. Deve-se se considerar também que neste período as condições climáticas são favoráveis a atividade pesqueira, proporcionando uma maior captura.

Das 15 espécies capturadas, quatro são estuarinas (Mugil platanus, Paralichthys orbignyanus, Micropogonias furnieri e Odontesthes argentinensis). Loebmann e Vieira (2005) estudando a distribuição 
TABELA 2: Freqüência absoluta (FA) e Freqüência relativa (FR) por estação do ano, freqüência absoluta total no período (FAT) e freqüência relativa total do período (FRT) das espécies capturadas na Lagoa Pequena, entre março de 2005 a fevereiro de 2006.

\begin{tabular}{|c|c|c|c|c|c|c|c|c|c|c|}
\hline \multirow{2}{*}{ Espécies/estação do ano } & \multicolumn{2}{|c|}{ Outono } & \multicolumn{2}{|c|}{ Inverno } & \multicolumn{2}{|c|}{ Primavera } & \multicolumn{2}{|c|}{ Verão } & \multicolumn{2}{|c|}{ Totais } \\
\hline & FA & FR & FA & FR & FA & FR & FA & FR & FAT & FRT \\
\hline \multicolumn{11}{|l|}{ Characiformes } \\
\hline Oligosarcus jenynsii & 4 & 7,2 & 6 & 9,5 & 8 & 6,8 & 10 & 10,5 & 28 & 8,5 \\
\hline Oligosarcus robustus & 4 & 7,2 & 6 & 9,5 & 11 & 9,4 & 7 & 7,4 & 28 & 8,5 \\
\hline Cyphocarax voga & 6 & 10,9 & 5 & 7,9 & 10 & 8,5 & 10 & 10,5 & 31 & 9,4 \\
\hline Hoplias malabaricus & 10 & 18,1 & 12 & 19,2 & 13 & 11,3 & 15 & 15,7 & 50 & 15,2 \\
\hline \multicolumn{11}{|l|}{ Perciformes } \\
\hline Geophagus brasiliensis & 2 & 3,6 & 5 & 7,9 & 6 & 5,1 & 7 & 7,4 & 20 & 6,1 \\
\hline Crenicichla punctata & 2 & 3,6 & 2 & 3,1 & 6 & 5,1 & 9 & 9,5 & 19 & 5,8 \\
\hline Crenicichla punctata & 2 & 3,6 & 2 & 3,1 & 6 & 5,1 & 9 & 9,5 & 19 & 5,8 \\
\hline Micropogonias furnieri & 1 & 1,8 & 1 & 1,6 & 5 & 4,3 & 1 & 1,1 & 8 & 2,4 \\
\hline \multicolumn{11}{|l|}{ Siluriformes } \\
\hline Hypostomus commersonii & 3 & 5,4 & 1 & 1,6 & 3 & 2,6 & 4 & 4,2 & 11 & 3,3 \\
\hline Loricariichthys anus & 5 & 9,4 & 6 & 9,5 & 9 & 7,7 & 9 & 9,5 & 29 & 8,8 \\
\hline Pimelodus maculatus & 4 & 7,2 & 5 & 7,9 & 10 & 8,5 & 4 & 4,2 & 23 & 7 \\
\hline Rhamdia aff. quelen & 5 & 9,4 & 5 & 7,9 & 9 & 7,7 & 5 & 5,3 & 24 & 7,3 \\
\hline \multicolumn{11}{|l|}{ Atheriniformes } \\
\hline Odontesthes bonariensis & 1 & 1,8 & 7 & 11,3 & 6 & 6,8 & 0 & 0 & 12 & 4,8 \\
\hline Odontesthes argentinensis & 4 & 7,2 & 0 & 0 & 5 & 4,3 & 4 & 4,2 & 13 & 3,9 \\
\hline \multicolumn{11}{|l|}{ Mugiliformes } \\
\hline Mugil platanus & 0 & 0 & 2 & 3,1 & 8 & 6,8 & 8 & 8,4 & 18 & 5,5 \\
\hline \multicolumn{11}{|l|}{ Pleuronectiformes } \\
\hline Paralichthys orbignyanus & 4 & 7,2 & 0 & 0 & 6 & 5,1 & 2 & 2,1 & 12 & 3,6 \\
\hline Total & 64 & 100 & 51 & 100 & 108 & 100 & 107 & 100 & 330 & 100 \\
\hline
\end{tabular}

Deve-se se considerar também que neste período as condições climáticas são favoráveis a atividade pesqueira, proporcionando uma maior captura.

e abundância de peixes na Lagoa do Peixe justifica o menor numero de espécies estuarino-residentes, pelo fato de que poucos animais conseguem desenvolver mecanismos fisiológicos para viverem em ambientes que apresentam grandes variações físicas.

Dentre as espécies de água doce houve uma predominância da traíra (Hoplias malabaricus) que representou $15,2 \%$ do total capturado. A espécie estuarina com maior freqüência de ocorrência foi a tainha (Mugil platanus) com 5,5\% (Tabela 2).

Quanto à biomassa, as espécies estuarino dependentes representaram $16,1 \%$ da biomassa total e as espécies de água doce $83,9 \%$. A traíra foi a espécie mais importante, representando $23,8 \%$ do total capturado (Tabela 3), isto pode ser atribuído ao fato desta espécie ser a de maior interesse econômico, de maneira que sobre ela é desenvolvido maior esforço de pesca. Silvano e Begossi (2001) estudando a pesca no Rio Piracicaba e Cetra e Petrere (2001), no Rio Tocantins, descrevem estratégias usadas por pescadores artesanais na busca por espécies de maior interesse econômico. Maccord et al. (2007) afirmam que pescadores artesanais da região amazônica comercializam espécies de maior valor econômico e as espécies de menor valor são utilizadas para consumo da família, sendo a nosso ver, a mesma estratégia utilizada pelos pescadores que atuam na Lagoa Pequena.

O tamanho de malha utilizado no local, não cumpre a legislação que regula a pesca na região estuarina, a qual estabelece um tamanho mínimo de $100 \mathrm{~mm}$ de malha de rede, o que tem como conseqüência a captura de animais de tamanho reduzido (Tabela 3 ). 
TABELA 3: Tamanho médio, número total, biomassa e percentual em biomassa (\%B) dos exemplares das espécies capturadas na Lagoa Pequena entre março de 2005 a fevereiro de 2006.

\begin{tabular}{|c|c|c|c|c|c|}
\hline Ordem/Espécies & $\begin{array}{c}\text { Comprimento Total } \\
\text { Médio }(\mathrm{cm})\end{array}$ & $\begin{array}{l}\text { Peso médio } \\
\text { (g) }\end{array}$ & $\mathrm{N}^{0}$ total & Biomassa (kg) & $\% \mathrm{~B}$ \\
\hline \multicolumn{6}{|l|}{ Characiformes } \\
\hline Oligosarcus jenynsii & 22,0 & 230 & 28 & 6,44 & 7,3 \\
\hline Oligosarcus robustus & 23,5 & 251 & 28 & 7,03 & 8 \\
\hline Cyphocarax voga & 14,7 & 40,4 & 31 & 1,25 & 1,4 \\
\hline Hoplias malabaricus & 13,0 & 420 & 50 & 21 & 23,8 \\
\hline \multicolumn{6}{|l|}{ Perciformes } \\
\hline Geophagus brasiliensis & 11,5 & 36 & 20 & 0,72 & 0,8 \\
\hline Crenicichla punctata & 19,0 & 155 & 19 & 2,79 & 3,2 \\
\hline Micropogonias furnieri & 31,3 & 238 & 8 & 1,9 & 2,2 \\
\hline \multicolumn{6}{|l|}{ Siluriformes } \\
\hline Hypostomus commersoni & 32,2 & 394 & 11 & 4,33 & 4,9 \\
\hline Loricariichthys anus & 29,5 & 340 & 29 & 9,86 & 11,2 \\
\hline Pimelodus maculatus & 34,1 & 271 & 23 & 6,23 & 7,1 \\
\hline Rhamdia aff. quelen & 27,1 & 399 & 24 & 9,58 & 10,9 \\
\hline \multicolumn{6}{|l|}{ Atheriniformes } \\
\hline Odontesthes bonariensis & 27,5 & 290 & 16 & 4,64 & 5,3 \\
\hline Odontesthes argentinensis & 32,0 & 110 & 13 & 1,43 & 1,6 \\
\hline \multicolumn{6}{|l|}{ Mugiliformes } \\
\hline Mugil sp. & 39,0 & 382 & 18 & 6,88 & 7,8 \\
\hline \multicolumn{6}{|l|}{ Pleuronectiformes } \\
\hline Paralichthys orbignyanus & 34,1 & 350 & 12 & 4,2 & 4,8 \\
\hline Total & - & - & 330 & 88,28 & - \\
\hline
\end{tabular}

A análise de correlação mostra que apenas o peixe Odontesthes bonariensis, apresentou correlação negativa $(\mathrm{r}=-0,64 ; \mathrm{p}=0,048)$ com a temperatura, confirmando a afirmação de Brown e Fuentes (2005), de que o peixe-rei O. bonariensis é uma espécie de águas temperadas.

As demais espécies não foram afetadas por fatores ambientais. Assim as diferenças na freqüência de ocorrência das espécies em relação aos períodos do ano devem-se, principalmente aos aspectos biológicos destas espécies, relacionados aos seus processos nutricionais e reprodutivos, de maneira que as condições ambientais do local não estão se sobrepondo às funções biológicas das espécies.

Deve-se considerar também que, os resultados obtidos neste trabalho podem estar sendo determinados pelos tamanhos de malha e artes de pesca utilizadas, que são próprias para espécies de água doce.

As variações ambientais sazonais na Lagoa Pequena propiciam condições para a presença de espécies estuarinas, mas não limita a presença das espécies de água doce, fazendo com que ambos os grupos contribuam nas capturas locais ao longo de todo ano.

\section{Referências}

Bemvenuti, M. A.; Moresco, A. 2005. Peixes - Áreas de banhados e lagoas costeiras do extremo sul do Brasil. ABRH, Porto Alegre, Brasil, 63pp.

Brown, D. R.; Fuentes, C. M. 2005. Validation of daily increment deposition in otolths of the pejerrey (Odontesthes bonariensis). Revista de Investigaciones Pesqueras, 17: 35-42.

Cerdeira, R. G. P.; Ruffino, M. L.; Isaac, V. J. 2000. Fish catches among riverside communities around Lago Grande de Monte Alegre, Lower Amazon, Brazil. Fisheries Management \& Ecology, 7 (4): 355-374.

Cetra, M.; Petrere, M. 2001. River Tocantins small-scale fisheries. Fisheries Management and Ecology, 8: 153-162.

Fischer, L. G.; Pereira, L. E. D.; Vieira, J. P. 2004. Peixes estuarinos e costeiros. Ecossistema, Rio Grande do Sul, Brasil, 127p.

Loebmann, D.; Vieira, J. P. 2005. Distribuição espacial e abundância das assembléias de peixes no Parque Nacional da Lagoa do Peixe, Rio Grande do Sul, Brasil. Revista Brasileira de Zoologia, 22 (3): 667-675. 
Loebmann, D.; Vieira, J. P.; Bemvenuti, M. A.; Astarloa, J. M. D.; Cousseau, M. B. Figueroa. D. 2008. Composição e abundância da icitofauna de duas laguinas costeiras da América do Sul Austral: lagoa do peixe $\left(31^{\circ} \mathrm{S} ; 51^{\circ} \mathrm{W} 0\right.$, Brasil e laguna Mar Chiquita $\left(37^{\circ} \mathrm{S}\right.$; $57^{\circ} \mathrm{W}$, Argentina. Neotropical Biology and Conservation, 3 (1): 28-33.

Garcia, A. M.; Vieira, J. P. 2001. O aumento da diversidade de peixes no estuário da Lagoa dos Patos durante o episódio El Niño 1997-1998. Atlântica, 23: 85-96.

ITEPA, Instituto de Pesquisa e Assessoria UCPel. 2002. Estrutura socioeconômica da atividade pesqueira no município de Pelotas. Informe socioeconômico $\mathrm{n}^{\circ}$ 7. Rio Grande do Sul, Brasil, 21pp.
Maccord, P. F. L.; Silvano, R. A. M.; Ramires, M. S.; Clauzet, M; Begossi, A. 2007. Dynamics of artisanal fisheries in two Brazilian Amazonian reserve: implications to co-management. Hydrobiologia, 583: 365-376.

Silvano, R. A. M.; Begossi, A. 2001. Seasonal dynamics of fishery at the Piracicaba River (Brazil). Fisheries Research, 51: 69-86.

Súarez, Y. R. 2008. Variação especial e temporal na diversidade e composição de espécies de peixes em riachos da bacia do Rio Ivinhema, Alto Rio Paraná. Biota Neotropica, 8 (3): 194-207.

Súarez, Y. R.; Juniorm M. P. 2005. Organização das assembléias de peixes em riachos da bacia do rio Iguatemi, Estado do Mato Grosso do Sul. Acta Scientiarum, 27 (2): 161-167. 